American Journal of Applied Sciences 6 (3): 396-400, 2009

ISSN 1546-9239

(C) 2009 Science Publications

\title{
Power Quality Improvement Using DVR
}

\author{
C. Benachaiba and B. Ferdi \\ Bechar University, Center BP, 417 Bechar 08000, Algeria
}

\begin{abstract}
Voltage sags and swells in the medium and low voltage distribution grid are considered to be the most frequent type of power quality problems based on recent power quality studies. Their impact on sensitive loads is severe. The impact ranges from load disruptions to substantial economic losses up to millions of dollars. Different solutions have been developed to protect sensitive loads against such disturbances but the DVR is considered to be the most efficient and effective solution. Its appeal includes lower cost, smaller size and its dynamic response to the disturbance. This research described DVR principles and voltage restoration methods for balanced and/or unbalanced voltage sags and swells in a distribution system. Simulation results were presented to illustrate and understand the performances of DVR under voltage sags/swells conditions.
\end{abstract}

Key words: Dynamic Voltage Restorer (DVR), voltage sags, voltage swells, power quality

\section{INTRODUCTION}

Dynamic voltage restores (DVRs) are now becoming more established in industry to reduce the impact of voltage dips on sensitive loads ${ }^{[1-3]}$. A voltage dip is commonly defined as any low voltage drop event between $10 \%$ and $90 \%$ of the nominal RMS voltage, lasting between 0.5 cycles and $1 \mathrm{~min}^{[4]}$. In comparison with interruptions, voltage dips affect a large number of customers and for some cases may cause extremely serious problems. Voltage dips are one of the most occurring power quality problems. They occur more often and cause severe problems and economical losses. There are different ways to mitigate voltage dips, swells and interruptions in transmission and distribution systems. At present, a wide range of very flexible controllers which capitalize on newly available power electronics components are emerging for custom power applications. Among these, the distribution static compensator and the dynamic voltage restorer are the most effective devices; both of them based on the voltage source converter (SVC) principle ${ }^{[5]}$.

Figure 1 shows a typical DVR series connected topology. The DVR essentially consists of a series inverter (VSI), inverter output filter and an energy storage device connected to the DC link.

The basic operation principle of the DVR is to inject an appropriate voltage in series with the supply through injection transformer whenever voltage sag or voltage swell is detected. In addition to voltage sags and swells compensation, DVR can also perform other

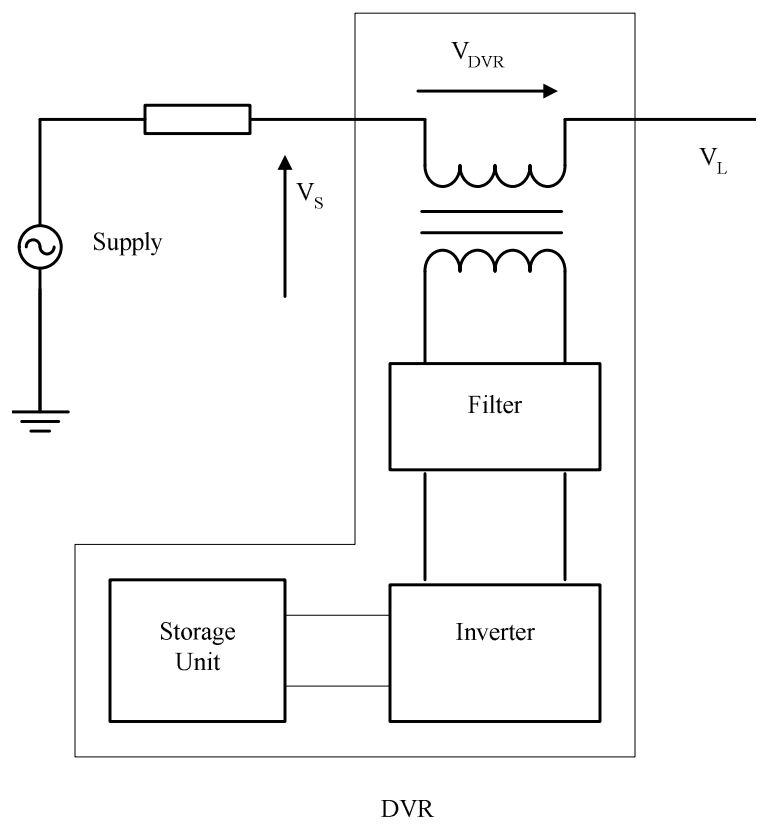

Fig. 1: DVR series connected topology

tasks such as harmonic compensation and Power Factor correction. Compared to the other Custom Power devices, the DVR clearly provides the best economic solution for its size and capabilities.

This research introduced Dynamic Voltage Restorer (DVR) and its voltage compensation methods. At the end, simulation results using MATLAB were illustrated and discussed.

Corresponding Author: C. Benachaiba, Bechar University, Center BP 417 Bechar 08000, Algeria 


\section{DYNAMIC VOLTAGE RESTORER}

A Dynamic Voltage Restorer (DVR) is a recently proposed series connected solid state device that injects voltage into the system in order to regulate the load side voltage. The DVR was first installed in $1996^{[6]}$. It is normally installed in a distribution system between the supply and the critical load feeder ${ }^{[7]}$. Its primary function is to rapidly boost up the load-side voltage in the event of a disturbance in order to avoid any power disruption to that $\operatorname{load}^{[8,9]}$. There are various circuit topologies and control schemes that can be used to implement a DVR. In addition to voltage sags and swells compensation, DVR can also perform other tasks such as: line voltage harmonics compensation, reduction of transients in voltage and fault current limitations.

The general configuration of the DVR consists of an Injection/Booster transformer, a Harmonic filter, a Voltage Source Converter (VSC), DC charging circuit and a Control and Protection system as shown in Fig. 1.

\section{CONVENTIONAL DVR VOLTAGE INJECTION METHODS}

The possibility of compensating voltage sag can be limited by a number of factors including finite DVR power rating, different load conditions and different types of voltage sag. Some loads are very sensitive to phase angle jump and others are tolerant to it. Therefore, the control strategy depends on the type of load characteristics. There are three distinguishing methods to inject DVR compensating voltage:

Pre-Dip Compensation (PDC): The PDC method tracks supply voltage continuously and compensates load voltage during fault to pre-fault condition. In this method, the load voltage can be restored ideally, but the injected active power cannot be controlled and it is determined by external conditions such as the type of faults and load conditions. The lack of the negative sequence detection in this method leads to the phaseoscillation in the case of single-line faults. Figure 2 shows the single-phase vector diagram of this method.

According to Fig. 2, the apparent power of DVR is:

$$
\begin{aligned}
\mathrm{S}_{\text {IDVR }} & =\mathrm{I}_{\mathrm{L}} \mathrm{V}_{\text {IDVR }} \\
& =\mathrm{I}_{\mathrm{L}} \sqrt{\mathrm{V}_{\mathrm{L}}^{2}+\mathrm{V}_{\mathrm{S}}^{2}-2 \mathrm{~V}_{\mathrm{L}} \mathrm{V}_{\mathrm{S}} \cos \left(\theta_{\mathrm{L}}-\theta_{\mathrm{S}}\right)}
\end{aligned}
$$

And the active power of DVR is:

$$
\mathrm{P}_{\mathrm{IDVR}}=\mathrm{I}_{\mathrm{L}}\left(\mathrm{V}_{\mathrm{L}} \cos \theta_{\mathrm{L}}-\mathrm{V}_{\mathrm{S}} \cos \theta_{\mathrm{S}}\right)
$$

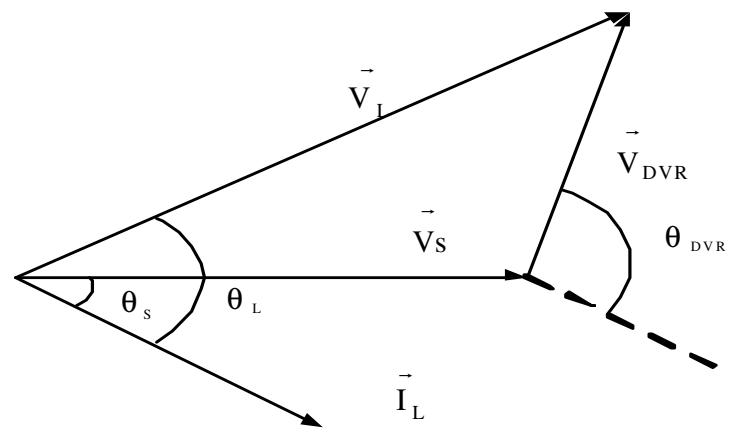

Fig. 2: Single-phase vector diagram of the PDC method

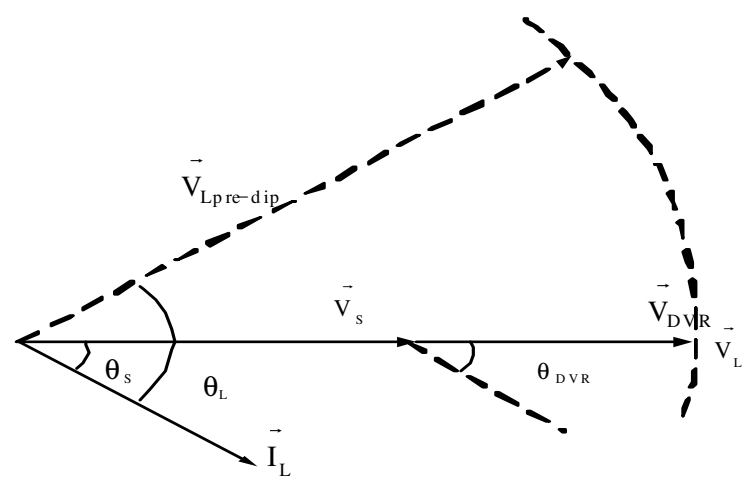

Fig. 3: Single-phase vector diagram of the IPC method

The magnitude and the angle of the DVR voltage are:

$$
\begin{gathered}
\mathrm{V}_{\mathrm{IDVR}}=\sqrt{\mathrm{V}_{\mathrm{L}}^{2}+\mathrm{V}_{\mathrm{S}}^{2}-2 \mathrm{~V}_{\mathrm{L}} \mathrm{V}_{\mathrm{S}} \cos \left(\theta_{\mathrm{L}}-\theta_{\mathrm{S}}\right)} \\
\theta_{\text {IDVR }}=\tan ^{-1}\left(\frac{\mathrm{V}_{\mathrm{L}} \sin \theta_{\mathrm{L}}-\mathrm{V}_{\mathrm{S}} \sin \theta_{\mathrm{S}}}{\mathrm{V}_{\mathrm{L}} \cos \theta_{\mathrm{L}}-\mathrm{V}_{\mathrm{S}} \cos \theta_{\mathrm{S}}}\right)
\end{gathered}
$$

In-Phase Compensation (IPC): This is the most used method in which the injected DVR voltage is in phase with the supply side voltage regardless of the load current and the pre-fault voltage as shown in Fig. 3. The IPC method is suitable for minimum voltage or minimum energy operation strategies ${ }^{[10]}$. In other word, this approach requires large amounts of real power to mitigate the voltage sag, which means a large energy storage device.

The apparent and active powers of DVR are:

$$
\begin{gathered}
\mathrm{S}_{2 \mathrm{DVR}}=\mathrm{I}_{\mathrm{L}} \mathrm{V}_{\mathrm{DVR}}=\mathrm{I}_{\mathrm{L}}\left(\mathrm{V}_{\mathrm{L}}-\mathrm{V}_{\mathrm{S}}\right) \\
\mathrm{P}_{2 \mathrm{DVR}}=\mathrm{I}_{\mathrm{L}} \mathrm{V}_{\mathrm{DVR}} \cos \theta_{\mathrm{S}}=\mathrm{I}_{\mathrm{L}}\left(\mathrm{V}_{\mathrm{L}}-\mathrm{V}_{\mathrm{S}}\right) \cos \theta_{\mathrm{S}}
\end{gathered}
$$


The magnitude and the angle of the DVR voltage are:

$$
\begin{aligned}
& \mathrm{V}_{2 \mathrm{DVR}}=\mathrm{V}_{\mathrm{L}}-\mathrm{V}_{\mathrm{S}} \\
& \theta_{2 \mathrm{DVR}}=\theta_{\mathrm{S}}
\end{aligned}
$$

In-Phase Advance Compensation (IPAC): Pre-Dip and in-phase compensation method must inject active power to loads to correct voltage disturbance. However, the amount of possible injection active power is confined to the stored energy in DC link, which is one of the most expensive components in DVR. Due to the limit of energy storage capacity of DC link, the DVR restoration time and performance are confined in these methods.

For the sake of controlling injection energy, in phase advance compensation method was proposed. The injection active power is made zero by means of having the injection voltage phasor perpendicular to the load current phasor. This method can reduce the consumption of energy stored in DC link by injecting reactive power instead of active power. Reducing energy consumption means that ride-through ability is increased when the energy storage capacity is fixed. On the other hand, the injection voltage magnitude of inphase advance compensation method is larger than those of pre-dip or in-phase compensation methods and the voltage phase shift can cause voltage waveform discontinuity, inaccurate zero crossing and load power swing. Therefore, in phase advance compensation method should be adjusted to the load that is tolerant to phase angle jump, or transition period should be taken while phase angle is moved from pre-fault angle to advance angle.

In short, IPAC method uses only reactive power and unfortunately, not all the sags can be mitigated without real power, as a consequence, this method is only suitable for a limited range of sags.

\section{SIMULATION}

In order to show the performance of the DVR in voltage sags and swells mitigation, a simple distribution network was simulated using MATLAB (Fig. 1). A DVR was connected to the system through a series transformer with a capability to insert a maximum voltage of $50 \%$ of the phase to ground system nominal voltage. In this simulation the In-Phase Compensation (IPC) method was used. The load considered in the study is a 5.5 MVA capacity with 0.92 p.f, lagging.

Voltage sags: A case of Three-phase voltage sag was simulated and the results are shown in Fig. 4. Figure 4a

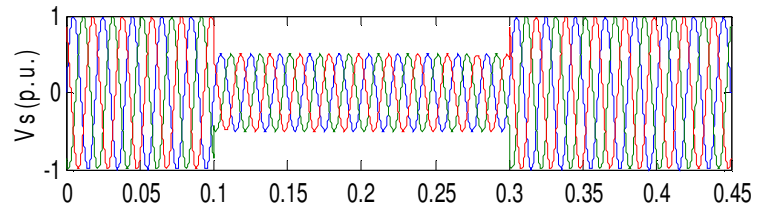

(a)

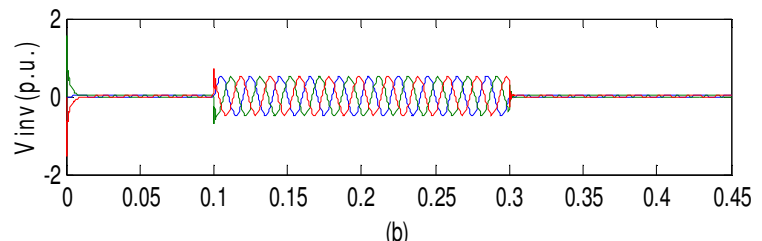

(b)

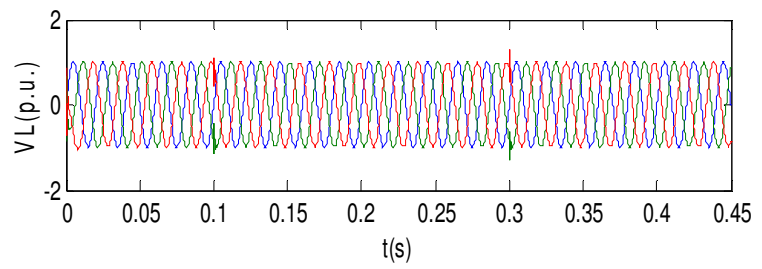

(c)

Fig. 4: Three-phase voltage sag; (a): Source voltages, (b): Injected voltages; (c): Load voltages

shows a 50\% voltage sag initiated at $100 \mathrm{~ms}$ and it is kept until $300 \mathrm{~ms}$, with total voltage sag duration of 200 $\mathrm{ms}$. Figure $4 \mathrm{~b}$ and $\mathrm{c}$ show the voltage injected by the DVR and the compensated load voltage, respectively. As a result of DVR, the load voltage is kept at 1 p.u. throughout the simulation, including the voltage sag period. Observe that during normal operation, the DVR is doing nothing. It quickly injects necessary voltage components to smooth the load voltage upon detecting voltage sag.

In order to understand the performance of the DVR under unbalanced conditions, Single-phase voltage sag was simulated and the results are shown in Fig. 5. The supply voltage with one phase voltage dropped down to $50 \%$ is shown in Fig. 5a. The DVR injected voltage and the load voltage are shown in Fig. 5b and c, respectively. As can be seen from the results, the DVR was able to produce the required voltage component rapidly and helped to maintain a balanced and constant load voltage at $1.00 \mathrm{p}$.u.

Voltage swells: The performance of DVR for a voltage swell condition was investigated. Here, the supply voltage swell was generated as shown in Fig. 6a. The supply three-phase voltage amplitudes were increased about $125 \%$ of nominal voltage. The injected threesphase voltage that was produced by DVR in order to correct the load voltage and the load voltage are shown in Fig. $6 \mathrm{~b}$ and c, respectively. As can be seen from the 


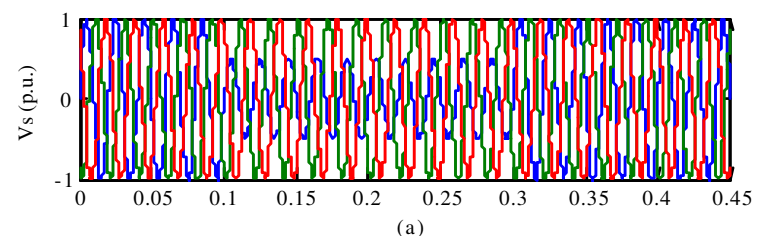

(a)

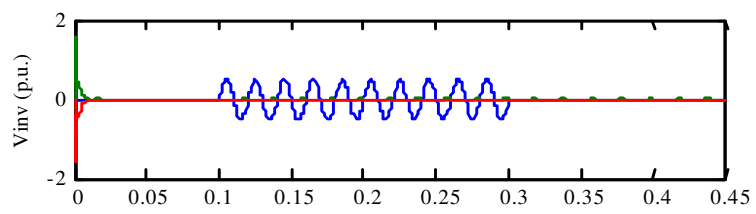

(b)

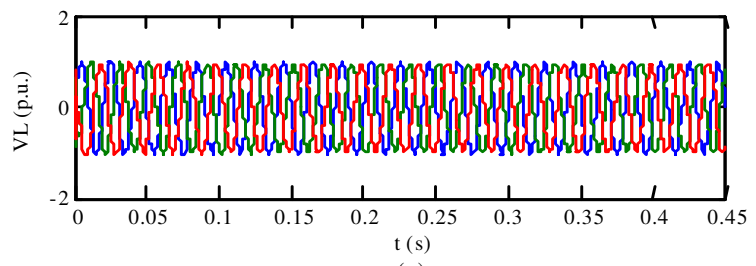

(c)

Fig. 5: Single-phase voltage sag; (a): Source voltages; (b): Injected voltage, (c): Load voltages
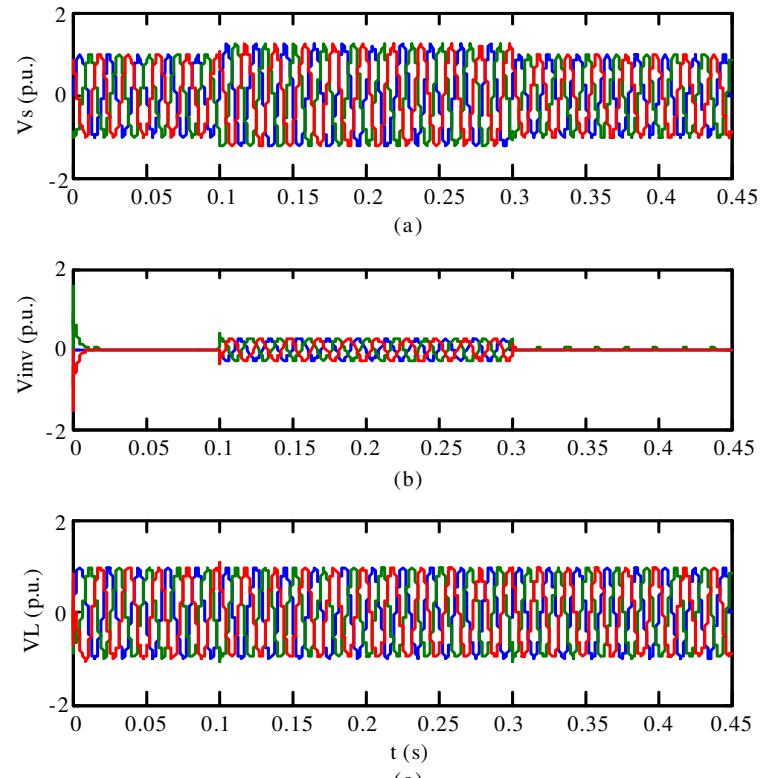

(c)

Fig. 6: Three-phase voltage swell; (a): Source voltages; (b): Injected voltages; (c): Load voltages

results, the load voltage was kept at the nominal value with the help of the DVR. Similar to the case of voltage sag, the DVR reacted quickly to inject the appropriate voltage component (negative voltage magnitude) to correct the supply voltage.
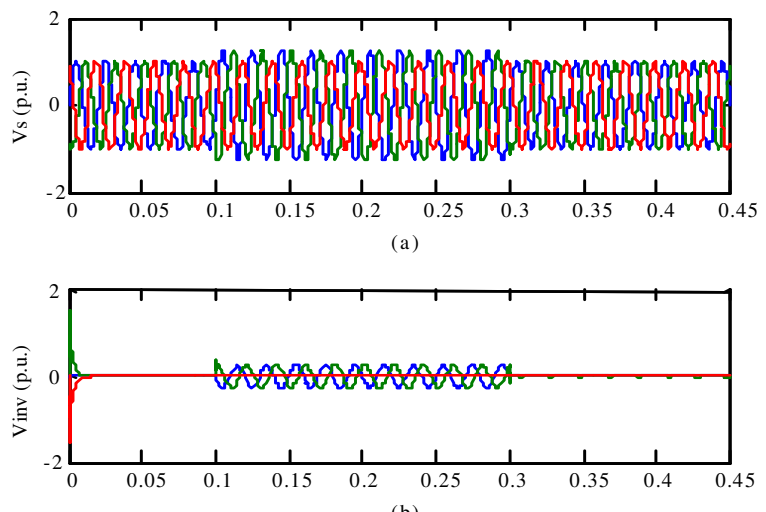

(b)

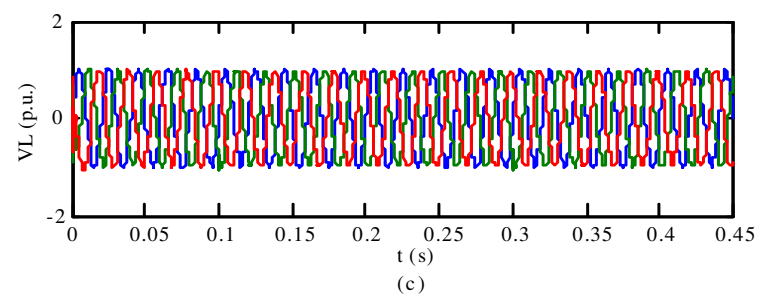

Fig. 7: Two-phase voltage swell; (a): Source voltages; (b): Injected voltages; (c): Load voltages

The performance of the DVR with an unbalanced voltage swell is shown in Fig. 7. In this case, two of the three phases are higher by $25 \%$ than the third phase as shown in Fig. 7a. The injected voltage that was produced by DVR in order to correct the load voltage and the load voltage are shown in Fig. $7 \mathrm{~b}$ and c, respectively. Notice the constant and balanced voltage at the load throughout the simulation, including during the unbalanced voltage swell event.

\section{CONCLUSION}

The simulation results showed clearly the performance of the DVR in mitigating voltage sags and swells. The DVR handled both balanced and unbalanced situations without any difficulties and injected the appropriate voltage component to correct rapidly any anomaly in the supply voltage to keep the load voltage balanced and constant at the nominal value. The efficiency and the effectiveness in voltage sags/swells compensation showed by the DVR makes him an interesting power quality device compared to other custom power devices.

\section{REFERENCES}

1. Ravi Kumar, S.V. and S. Siva Nagaraju, 2007. Power quality improvement using d-statcom and DVR. Int. J. Elect. Power Eng., 1: 368-376. 
2. Nielsen, J.G., 2002. Design and control of a dynamic voltage restorer. Ph. D. Thesis, Aalborg University, Institute of Energy Technology. ISBN: 87-89179-42-0.

3. John Newman, M., D. Grahame Holmes, J. Godsk Nielsen and F. Blaabjerg, 2003. A Dynamic Voltage Restorer (DVR) with selective harmonic compensation at medium voltage level. IEEE.

4. Moreno-Muñoz, A., D. Oterino, M. González and F.A. Olivencia, 2006. Study of Sag Compensation with DVR: Benalmádena (Málaga), Spain. IEEE MELECON May, pp: 16-19.

5. Stump, M.D., G.J. Kaene and F.K.S. Leong, 1998. Role of custom power products in enhancing power quality at industrial facilities. In: Conf. Rec. IEEE/EMPD, pp: 507-517.

6. Li, B.H., S.S. Choi and D.M. Vilathgamuwa, 2001. Design considerations on the line-side filter used in the dynamic voltage restorer: IEE Proc. Generat. Transmission Distribut., 148: 1-7.
7. Chan, K., 1998. Technical and performance aspects of a dynamic voltage restorer. In IEE Half Day Colloquium on Dynamic Voltage RestorersReplacing Those Missing Cycles, pp: 5/1-525.

8. Buxton, R., 1998. Protection from voltage dips with the dynamic voltage restorer. In IEE Half Day Colloquium on Dynamic Voltage RestorersReplacing Those Missing Cycles, pp: 3/1-3/6.

9. Sng, E.K.K., S.S. Choi and D.M. Vilathga-Muwa, 2004. Analysis of series compensation and DC-link voltage controls of a transformerless self-charging dynamic voltage restorer. IEEE Trans. Power Delivery, 19: 1511-1518.

10. E-Otadui, U. Viscarret, S. Bacha, M. Caballero and R. Reyero, 2002. Evaluation of different strategies for series voltage sag cornpensation. Proc. IEEE PESC, 4: 1797-1802. 